\title{
Evaluation de la production du Moringa oleifera Lam. sur substrat constitué de boue de vidange en fonction de la fertilisation et de la dose d'irrigation
}

\author{
Ibrahim SOUMAÏLA SIDDO ${ }^{1 *}$, Mahaman Moustapha ADAMOU ${ }^{1}$, \\ Abdourahmane TANKARI DAN BADJO ${ }^{2}$ et Zaleyha AMADOU BANA ${ }^{1}$ \\ ${ }^{1}$ Département Genie Rural/Eaux et Forêts/Faculté d'Agronomie, Université Abdou Moumouni, BP: 10960 \\ Niamey, Niger. \\ ${ }^{2}$ Département Sciences du sol/ Faculté d'Agronomie, Université Abdou Moumouni, BP: 10960 Niamey, Niger. \\ *Auteur correspondant ; E-mail : siddo855@gmail.com; Tél. : (+227) 995655 14/91 330339
}

Received: 27-07-2021

\section{RÉSUMÉ}

Dans le contexte de développement durable, la gestion des déchets, quelle que soit leur nature occupe une place importante. La non valorisation des boues de la Société d'Exploitation des Eaux du Niger (SEEN) a motivé la réalisation de cette étude. Menée en condition semis contrôlée, elle avait pour objectif d'évaluer la production du Moringa oleifera sur boue de vidange en fonction de deux doses d'irrigation combinées à différents types de fertilisation. Le dispositif expérimental était un split plot à quatre répétitions et deux facteurs: la dose d'irrigation $(0,5 \mathrm{~L}$ et $1 \mathrm{~L})$ et la composition du substrat (B, BSC, BSE, BC, BS et S). Les paramètres observés sont: le taux de survie, la hauteur des plantules, le nombre de feuilles, le diamètre au collet de la plante, l'élongation racinaire et la biomasse totale. Les résultats ont montré que la dose d'irrigation de « 1 litre » a été plus favorable sur l'ensemble des substrats et que les plants issus des substrats Boue + Sol ; Boue + Sol + Engrais et Boue + Sol + Compost ont donné les meilleurs résultats. Par contre, une forte létalité des plantes sur les substrats composés uniquement de Boue a été observée. Cette boue pourrait être utilisée en agriculture à condition qu'elle soit supplémentée par des fertilisants organiques.

(C) 2021 International Formulae Group. All rights reserved.

Mots clés : Boue de vidange, Moringa oleifera, irrigation, fertilisation, Niger.

\section{Evaluation of the production of Moringa oleifera Lam. on the substrate based on mud of drain according to the fertilization and the dose of irrigation}

\begin{abstract}
In the context of durable context of development, the management of the waste, whatever the nature has an important place. The non-valorisation of the mud of the Niger Society of Exploitation of Water (NSEW) motivated the realization of this research leaded in seedling controlled condition. The research objective is evaluate the production of Moringa oleifera on the mud of drain according to two doses of irrigation combined on different types of fertilization. The experimental device was Split plot of four repetitions and the two factors: the dose of $(0.5 \mathrm{~L}$ and $1 \mathrm{~L})$ and the composition of the substrate $(\mathrm{B}, \mathrm{BSC}$ BSE, BC and S). The parameters observed are the rate of survival, the height of the seedling, the number of leaves, the diameter collar of the seedling, the elongation of the roots and the total biomass. The results have showed that the dose of irrigation of
\end{abstract}


$1 \mathrm{~L}$ was more favorable on the whole substractes whereas the seeds of substrates mud + Sol, mud + sol + fertilizer and mud + sol + compost had given the best results although a Big lethality of the seedling on the substractes composed only of mud was observed. This mud could be used in agriculture in condition that it may be followed by some organic fertilizers.

(C) 2021 International Formulae Group. All rights reserved.

Keywords: Mud of drain, Moringa oleifera, irrigation, fertilization, Niger.

\section{INTRODUCTION}

De nos jours, la production des déchets a augmenté de façon considérable malgré les efforts de prévention (Vorburger, 2006). La gestion de ce "problème de déchets » est un facteur essentiel dans une politique de développement durable (Morana, 2005). Les divers modes de gestion des déchets ainsi que le réemploi ou la réutilisation suscitent de multiples interrogations sur les impacts environnementaux et sanitaires qu'ils seraient susceptibles de présenter (CUSSTR, 2008; ADEME, 2011; AMORCE, 2012). L'élimination et la maîtrise des boues, longtemps considérées comme un aspect secondaire de la dépollution des eaux acquiert désormais une importance nouvelle à l'image du recyclage des déchets (Pisson, 2000). Les différentes voies de valorisation et d'élimination des boues sont l'épandage, la méthanisation, le compostage, l'incinération et la mise en décharge (Farid et Sonia, 2016). Au Niger, la production de l'eau potable à partir des eaux du fleuve engendre des quantités importantes de boues qui posent le problème de leur devenir. Cependant, le moyen le plus adéquat pour l'élimination des boues résiduaires serait leur valorisation agricole (Igoud, 2001), qui constitue un mode de gestion plus rationnel (APRIFEL, 2001) et contribue à une réintégration des éléments minéraux et organiques dans les sols (Chouial et al., 2017). Le maraîchage étant une exploitation continue du sol, l'utilisation des boues contribuerait à restaurer les éléments exportés par les cultures. Le Moringa, cultivé aux alentours des bas-fonds et du fleuve Niger (Djibo et al., 2017), joue un rôle socioéconomique important (Foidl et al., 2001 ; Anwar et Bhanger, 2003 ; Bosch, 2011 ; Madi et al., 2012 ; Abasse et al., 2017). D'où l'importance de ce travail qui portait sur l'évaluation de la production du Moringa oleifera Lam. sur substrat constitué de boue de vidange en fonction de la fertilisation et de la dose d'irrigation.

\section{MATERIEL ET METHODES}

L'étude a été réalisée à Niamey (République du Niger), précisément sur le site expérimental de la Faculté d'Agronomie de l'Université Abdou Moumouni. Il est situé à $13^{\circ} 29^{\prime} 59,15^{\prime}$ ' latitude Nord et $2^{\circ} 05^{\prime} 23,75^{\prime}$ ' longitude Est sous un climat de type Sudsahélien. Le matériel végétal utilisé est la variété PKM-1 du Moringa oleifera Lam. Des plantules obtenues, dix-huit jours (18) après semis, par germination des amandes scarifiées en pépinières, ont été repiquées dans des pots en plastique de $25,5 \mathrm{~cm}$ de long, $30 \mathrm{~cm}$ de diamètre et 12 litres de volume. Les substrats se composaient de la boue de vidange (boue issue du traitement de l'eau potable) collectée à la Société d'Exploitation des Eaux du Niger $(\mathrm{SEEN})$; du sol prélevé à la Faculté d'Agronomie; de l'engrais chimique (NPK: 15-15-15) provenant de la Centrale d'Approvisionnement en Intrants et Matériels Agricoles (CAIMA) et du compost provenant de la Fédération des Coopératives Maraîchères du Niger (FCMN-Niya) (Photo1 et 2).

\section{Dispositif expérimental}

Le dispositif expérimental utilisé est un split plot à quatre (4) répétitions et deux facteurs : facteur irrigation et facteur substrat. Le facteur irrigation a deux niveaux : $1 \mathrm{~L}$ et 0,5 L pour une fréquence d'irrigation de 48 heures. Le facteur substrat comprend six (6) types: $100 \%$ Boue (B) ; 50\% Boue $+30 \%$ Sol $+20 \%$ Compost (BSC); $50 \%$ Boue $+50 \%$ Sol $+2 \mathrm{~g}$ d'Engrais (BSE); 50\% Boue + 50\% Compost (BC) ; $80 \%$ Boue $+20 \%$ Sol (BS) et $100 \%$ Sol pour le témoin (S) (Photo 13). Chaque grande 
parcelle comprend six (6) traitements repartis de manière aléatoire, ce qui correspond à 12 traitements par répétition (Figure 1). Ensuite, au bout de deux (2) mois, les paramètres de croissance à savoir: la hauteur aérienne, le diamètre, et le nombre des feuilles ont été mesurés chaque semaine à l'aide d'un décamètre et d'un pied à coulisse. La dernière étape du suivi a consisté à déterrer les plants afin de peser le poids frais et de procéder au séchage à l'étuve pour évaluer le rapport de la biomasse sèche des plants (Photo 3 et Photo 4).

\section{Traitement des données}

Le logiciel Gestalt version 9th Edition a été utilisé pour l'analyse de la variance. Lorsque l'hypothèse nulle était rejetée au seuil de $5 \%$, la comparaison des moyennes avec le test de Newman-Kells était effectuée. Le tableur Excel de Microsoft version 2007 a servi à la réalisation des graphiques et tableaux.

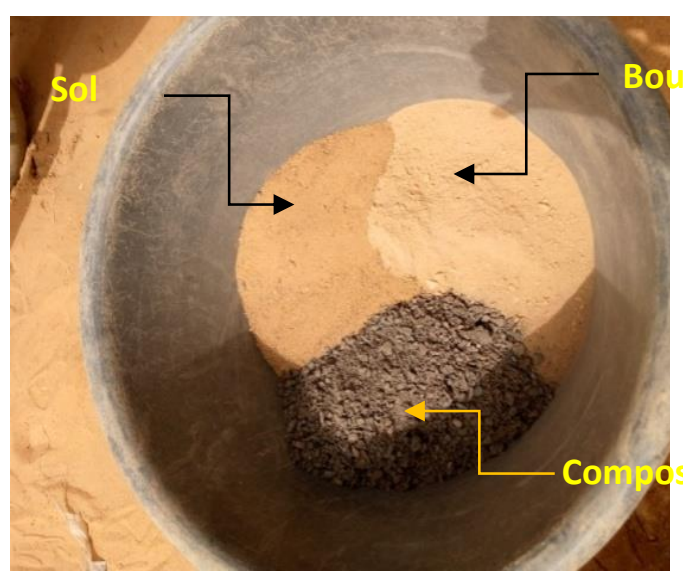

Photo 1 : Les trois composantes des substrats.

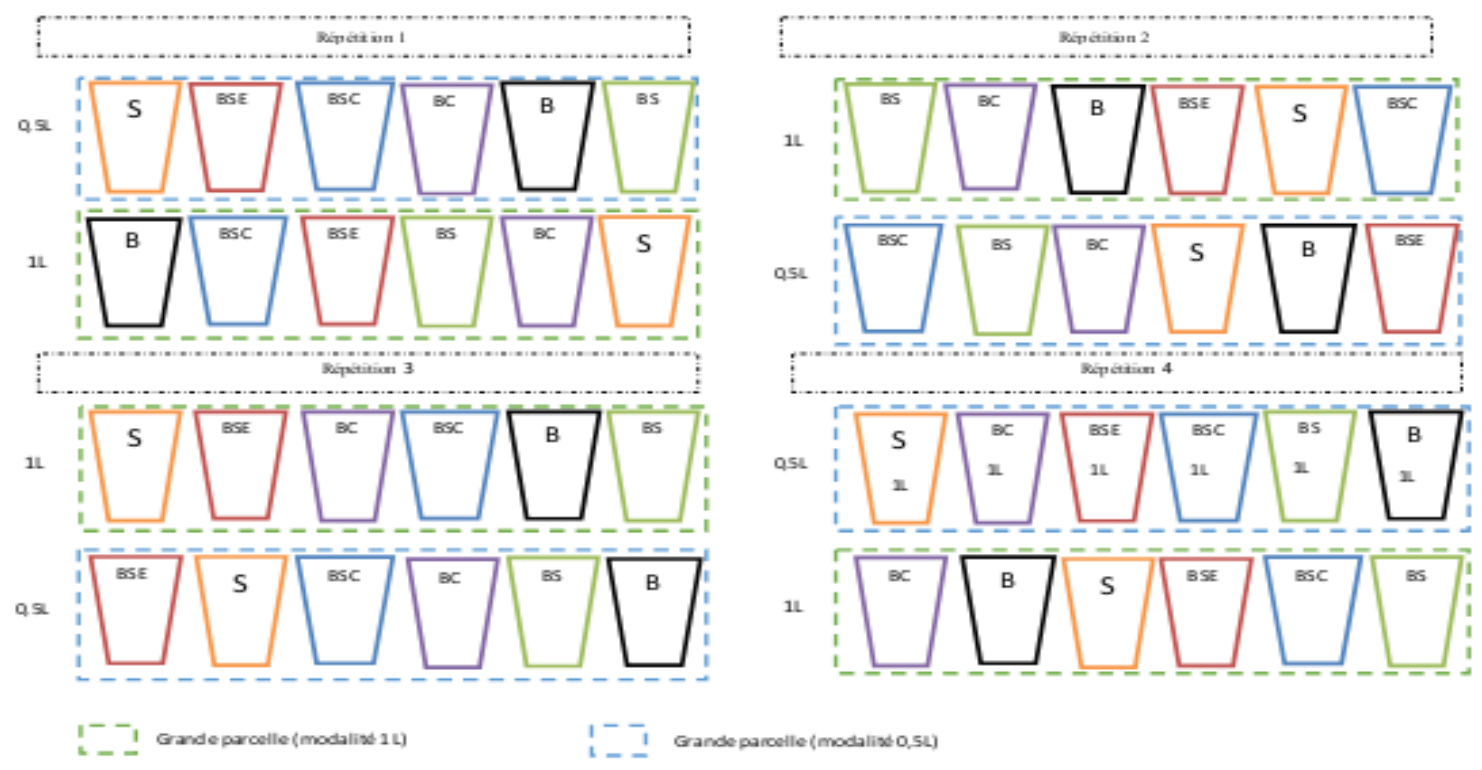

Figure 1: Schéma du dispositif expérimental.

B: Boue, BC : Boue + Compost, BS : Boue + Sol, BSC : Boue+ Sol+ Compost, BSE : Boue+ Sol +Engrais, S : Sol témoin.

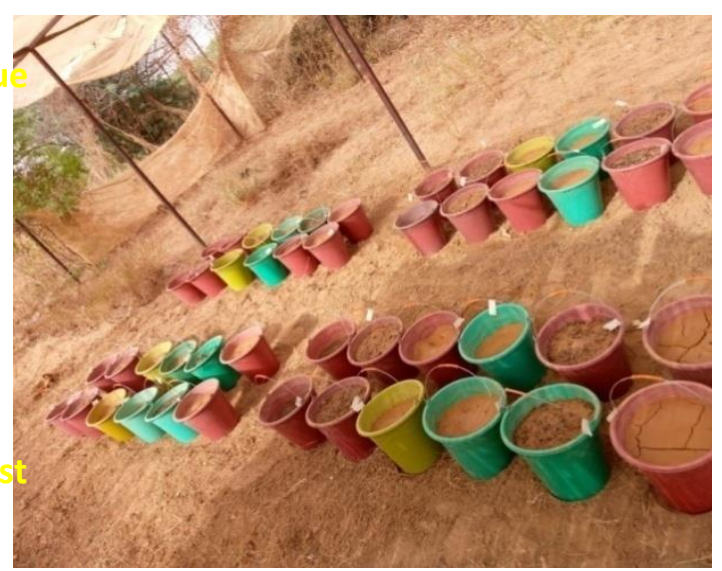

Photo 2 : Pots remplis des substrats. 


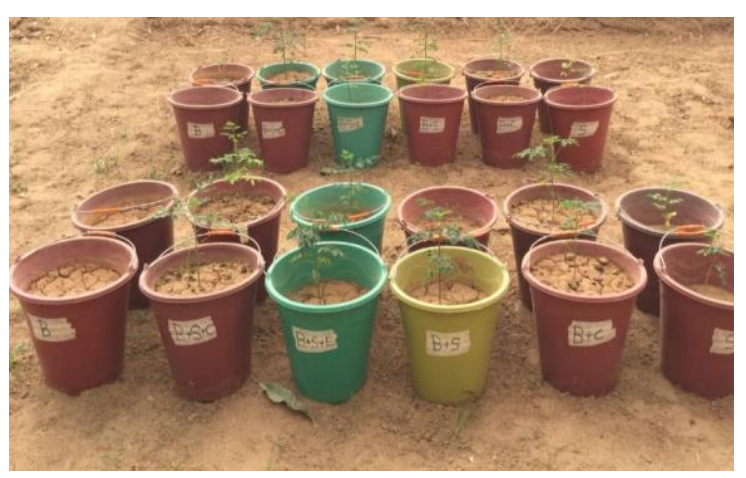

Photo 3 : Plantes avant la récolte.

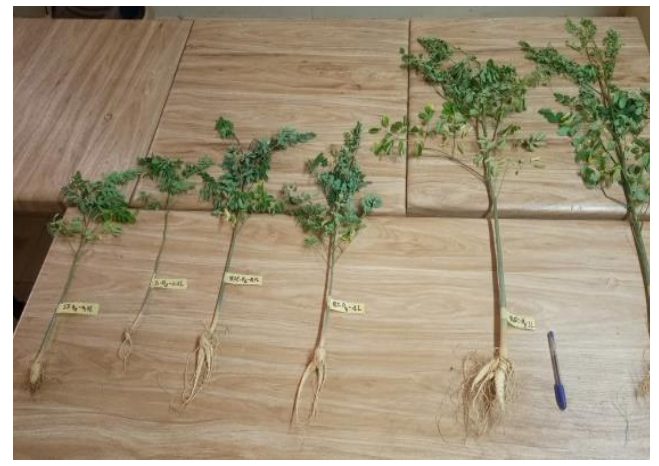

Photo 4 : Plantes après la récolte.

\section{RESULTATS}

\section{Taux de survie des plantes en fonction des substrats et de la dose d'irrigation}

La Figure 2 illustre le taux de survie des plantes en fonction des différents types de substrats et de la dose d'irrigation. On constate que pour la dose d'irrigation de $0,5 \mathrm{~L}$, les substrats BSE et BS ont présenté chacun un taux de survie de $100 \%$, suivis du substrat BSC ayant enregistré un taux de $75 \%$, et les substrats $\mathrm{BC}$ et $\mathrm{S}$ ont présenté chacun un taux de survie de $50 \%$. Par contre pour la dose d'irrigation de 1L, c'étaient les substrats BSC, BSE et S qui ont présenté chacun un taux de survie de $100 \%$, suivis des substrats BC et BS avec de taux de survie respectifs de $75 \%$ et $50 \%$ et le substrat B avec un taux de survie de $25 \%$. Les substrats BSE et BSC étaient les plus favorables à la survie des plantes quel qu'en soit la dose d'irrigation contrairement au substrat B qui a demeuré le moins favorable avec de taux respectifs de 0 et $25 \%$ pour les doses d'irrigation de 0,5 et $1 \mathrm{~L}$.

Notons qu'en raison de la forte létalité des plantes sur les substrats composés uniquement de boue (Figure 2), ces dernières n'ont pas fait l'objet d'analyse des paramètres phénologiques et de rendement.

\section{Accroissement en hauteur et diamètre}

L'analyse statistique des paramètres de croissance après deux (2) mois d'observation des plantes a montré des différences significatives entre ces paramètres, tant en fonction des substrats $(\mathrm{P}<0,007)$ que les doses d'irrigation appliquées $(P<0,001)$. C'est ainsi que, le substrat BS ayant reçu la dose d'irrigation de 1 L était statistiquement meilleur que tous les types de substrats ayant reçu 0,5 L. Cependant, il n'était pas supérieur à ces mêmes substrats à la dose de $1 \mathrm{~L}$, exceptée le témoin $\mathrm{S}$. Les diamètres des plantes issues du substrat BS étaient aussi supérieurs à ceux des substrats BSC et S mais, ils n'étaient pas tous différents des autres traitements (Tableau 1). L'accroissement de ces paramètres a été favorisé par les substrats BS, BSC et $\mathrm{BSE}$ avec des valeurs respectives de $\mathrm{H}=$ $40,75 \pm 11,26 \mathrm{~cm}$ et $\mathrm{d}=0,91 \pm 0,22 \mathrm{~cm}$; $\mathrm{H}=32,82 \pm 10,45 \mathrm{~cm}$ et $\mathrm{d}=0,75 \pm 0,21 \mathrm{~cm}$; $\mathrm{H}=31,38 \pm 9,78 \mathrm{~cm}$ et $\mathrm{d}=0,78 \pm 0,04 \mathrm{~cm}$. Enfin, la dose d'irrigation de $1 \mathrm{~L}$ a permis une meilleure croissance des plantes en hauteur comme en diamètre sur tous les substrats, excepté le témoin.

\section{Multiplication du nombre de feuilles et l'élongation racinaire}

Pour l'ensemble des substrats, c'était avec la dose d'irrigation de $1 \mathrm{~L}$ que les plus grands nombres de feuilles ont été enregistrés (Tableau 2). En ce qui concerne la dose $0.5 \mathrm{~L}$, les substrats $\mathrm{BS}$ et $\mathrm{BC}$ ont donné les nombres élevés de feuilles comparativement aux BS et BSE pour la dose de $1 \mathrm{~L}$. La différence était significative entre les doses d'irrigation $(\mathrm{P}<$ $0,007)$ pour ce paramètre. Pour l'élongation racinaire, bien que les différences n'étaient 
significatives, le substrat sol a plus favorisé le développement racinaire avec les deux doses d'irrigation.

\section{Biomasses totales fraiche et sèche}

La comparaison des moyennes des biomasses fraiches par le test de Newman keuils au seuil de 5\% n'a pas mis pas en évidence une différence significative entre les substrats $(P<0,212)$. Cette différence était significative entre les doses d'irrigation $(\mathrm{P}<$ 0,007). Par rapport aux biomasses sèches, la différence n'était ni significative entre les substrats $(P<0,174)$ ni significative entre les doses d'irrigation $(\mathrm{P}<0,024)$. De ce fait, les substrats BC et BSE semblent être les meilleurs avec la dose de 0,5 L. Tandis qu'avec la dose de $1 \mathrm{~L}$, c'étaient les substrats BS et BSC qui ont enregistré les meilleurs poids en termes de biomasses fraiches (Tableau 3). Sur tous les substrats, la dose d'irrigation de $1 \mathrm{~L}$ a plus favorisé le développement des plantes par rapport à celle de $0,5 \mathrm{~L}$. Après séchage des différentes parties végétales (tiges, feuilles et racines), c'étaient toujours les substrats BS et BSC qui ont enregistré les meilleurs poids de biomasses avec la dose de $1 \mathrm{~L}$ et $\mathrm{BS}$, BSC avec celle de $0,5 \mathrm{~L}$.

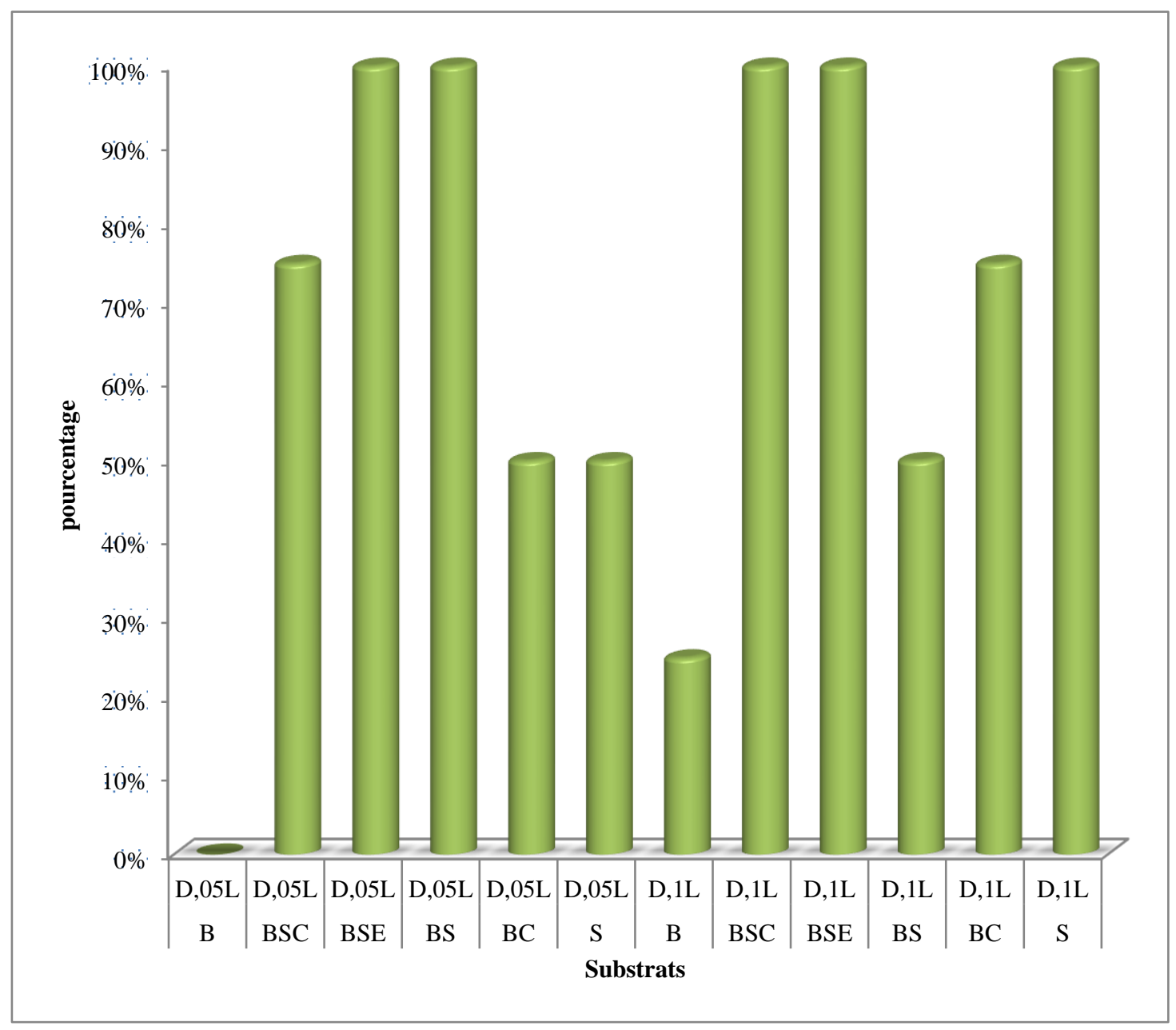

Figure 2: Taux de survie des plantes en fonction des substrats et de la dose d'irrigation. 
Tableau 1 : Accroissement en hauteur et en diamètre.

\begin{tabular}{l|llll}
\hline Substrats & Hauteur $(\mathbf{c m})$ & Diamètre $(\mathbf{c m})$ & \\
\hline & D.05 L & D.1 L & D.05 L & D.1 L \\
BC & $23,75 \pm 0,29^{\mathrm{a}}$ & $28,83 \pm 12,81^{\mathrm{ab}}$ & $0,66 \pm 0,05^{\mathrm{ab}}$ & $0,68 \pm 0,14^{\mathrm{ab}}$ \\
BS & $22,875 \pm 5,62^{\mathrm{a}}$ & $40,75 \pm 11,26 \mathrm{~b}$ & $0,63 \pm 0,14^{\mathrm{ab}}$ & $0,91 \pm 0,22^{\mathrm{bc}}$ \\
BSC & $19,83 \pm 3,52^{\mathrm{a}}$ & $32,82 \pm 10,45^{\mathrm{ab}}$ & $0,55 \pm 0,12 \mathrm{a}$ & $0,75 \pm 0,21^{\mathrm{ab}}$ \\
BSE & $21,87 \pm 4,92^{\mathrm{a}}$ & $31,38 \pm 9,78 \mathrm{ab}$ & $0,64 \pm 0,12^{\mathrm{ab}}$ & $0,78 \pm 0,04^{\mathrm{ab}}$ \\
S & $18,25 \pm 2,02^{\mathrm{a}}$ & $15,88 \pm 1,49^{\mathrm{a}}$ & $0,63 \pm 0,08^{\mathrm{ab}}$ & $0,56 \pm 0,10^{\mathrm{a}}$ \\
\hline
\end{tabular}

B: Boue, BC : Boue + Compost, BS : Boue + Sol, BSC : Boue+ Sol+ Compost, BSE : Boue+ Sol +Engrais, S : Sol témoin, LSDDiamètre $=0,088$, LSDHauteur $=4,644$.

Tableau 2 : Nombre de feuilles et élongation racinaire.

\begin{tabular}{|c|c|c|c|c|}
\hline \multirow[t]{2}{*}{ Substrats } & \multicolumn{2}{|c|}{ Nombre de feuilles } & \multicolumn{2}{|c|}{ Elongation racinaire $(\mathbf{c m})$} \\
\hline & D.05 L & D. $1 \mathrm{~L}$ & D.05 L & D.1 L \\
\hline $\mathrm{BC}$ & $6,50 \pm 1,73^{\mathrm{a}}$ & $7,33 \pm 0,94^{\mathrm{a}}$ & $10,00 \pm 1,73^{\mathrm{a}}$ & $8,16 \pm 2,01^{\mathrm{a}}$ \\
\hline BS & $7,00 \pm 2,94^{\mathrm{a}}$ & $10,00 \pm 4,62^{\mathrm{a}}$ & $11,50 \pm 3,58^{\mathrm{a}}$ & $13,50 \pm 0,58^{\mathrm{a}}$ \\
\hline $\mathrm{BSC}$ & $4,67 \pm 2,05^{\mathrm{a}}$ & $7,25 \pm 1,71^{\mathrm{a}}$ & $11,90 \pm 3,25^{\mathrm{a}}$ & $10,38 \pm 0,95^{\mathrm{a}}$ \\
\hline BSE & $6,25 \pm 2,06^{\mathrm{a}}$ & $10,00 \pm 2,16^{\mathrm{a}}$ & $11,50 \pm 3,19^{\mathrm{a}}$ & $12,45 \pm 3,42^{\mathrm{a}}$ \\
\hline$S$ & $5,50 \pm 0,58^{\mathrm{a}}$ & $6,00 \pm 1,83^{\mathrm{a}}$ & $12,05 \pm 3,75^{\mathrm{a}}$ & $13,53 \pm 1,87^{a}$ \\
\hline
\end{tabular}

B : Boue, BC : Boue + Compost, BS : Boue + Sol, BS : Boue + Sol + Compost, BSE : Boue + Sol + Engrais, S : Sol témoin, $\mathrm{LSD}($ Nombre de feuilles $)=1,504, \mathrm{LSD}_{\text {Elongation racinaire }}=1,753$.

Tableau 3: Biomasses fraiche et sèche.

\begin{tabular}{|c|c|c|c|c|}
\hline \multirow[t]{2}{*}{ Substrats } & \multicolumn{2}{|c|}{ Biomasse fraiche (g) } & \multicolumn{2}{|c|}{ Biomasse sèche (g) } \\
\hline & D. $05 \mathrm{~L}$ & D. $1 \mathrm{~L}$ & D. $05 \mathrm{~L}$ & D. $1 \mathrm{~L}$ \\
\hline $\mathrm{BC}$ & $13,60 \pm 0,81^{\mathrm{a}}$ & $19,60 \pm 10,88^{a}$ & $3,30 \pm 0,35^{\mathrm{a}}$ & $4,10 \pm 2,32^{\mathrm{a}}$ \\
\hline BS & $10,65 \pm 8,17^{\mathrm{a}}$ & $31,90 \pm 22,98^{\mathrm{a}}$ & $2,12 \pm 1,86^{\mathrm{a}}$ & $5,90 \pm 4,27^{\mathrm{a}}$ \\
\hline BSC & $9,83 \pm 8,47^{\mathrm{a}}$ & $27,62 \pm 19,43^{\mathrm{a}}$ & $2,70 \pm 2,90^{\mathrm{a}}$ & $6,58 \pm 5,20^{a}$ \\
\hline BSE & $11,42 \pm 7,64^{\mathrm{a}}$ & $17,32 \pm 9,23^{\mathrm{a}}$ & $2,42 \pm 1,87^{\mathrm{a}}$ & $3,58 \pm 2,11^{\mathrm{a}}$ \\
\hline S & $7,60 \pm 3,35^{\mathrm{a}}$ & $8,57 \pm 3,48^{a}$ & $1,40 \pm 0,46^{\mathrm{a}}$ & $1,58 \pm 0,89^{\mathrm{a}}$ \\
\hline
\end{tabular}




\section{DISCUSSION}

Les substrats BS, BSE, BSC et S ont enregistré les meilleurs taux de survie et la dose d'irrigation de $1 \mathrm{~L}$ a été globalement meilleure pour tous les paramètres de rendement évalués. Cette influence est due à l'enrichissement et à l'amélioration de la structure de la boue par l'apport du sol, du compost et de l'engrais. En effet, l'apport de la fumure organique améliore la production de Moringa oelifera (Foidl et al., 2001 ; Amaglo et al., 2006; Malo, 2014 ; Haouvang et al., 2017). Ces résultats sont identiques à ceux trouvés par De-SaintSauveur et Broin (2010), Agroconsult (2016), Laurence (2017), qui ont montré que le Moringa peut certes pousser sur tous types de sols, mais ce sont surtout les terrains fertiles et bien drainés qui lui conviennent. Phatu (2019) a obtenu un meilleur rendement avec des récipients de $300 \mathrm{ml}$, plus petits que ceux qui ont été utilisés dans cette étude. Pour ce qui est de la réaction des plantes aux différents substrats, on constate que la croissance des plantes était meilleure sur les substrats BS, BSE et BSC pour la plupart des paramètres étudiés. Ce résultat corrobore ceux de Pamo et al. (2002), Yansoumba (2002) et Malo (2014) qui ont montré que le Moringa oleifera crôit plus après un apport de fertilisants. Par contre, les faibles valeurs des paramètres ont été observées sur les plantes cultivées sur des substrats composés uniquement de boue aux deux niveaux de doses d'irrigation. Cela s'expliquerait par le $\mathrm{pH}$ faiblement acide de la boue utilisée $(4,16)$, son faible taux de matière organique et sa texture argileuse. Ces résultats concordent avec ceux de Zakaria (2013) qui, en évaluant la production de Moringa oleifera en pot sur des substrats (argile et sable), a montré que les plants issus du traitement de l'argile ont eu des difficultés d'absorption d'eau du fait de leur forme compacte qui ne laisse pas s'infiltrer l'eau et l'air et qui empêche au système racinaire de se développer normalement. Bien qu'il puisse pousser sur un sol neutre ou légèrement acide, le Moringa préfère un $\mathrm{pH}$ compris entre 6,3 à 7,0 (Koul et Chase, 2015). Martin (2007) a obtenu une croissance impressionnante de Moringa oleifera sur un sol pauvre et alcalin en lui apportant du compost.
En plus, les biomasses aériennes obtenues dans le cadre de cette étaient plus élevées que les biomasses souterraines. Ce qui corrobore les résultats de Sattah et al. (2019) qui avaient travaillé sur la même espèce sous l'effet du plomb. Cette expérimentation indique que la boue peut avoir un effet bénéfique si elle est combinée avec d'autres types de fertilisants comme compost et engrais chimiques. Les effets à court terme étudiés indiquent que l'apport de la boue a eu un effet bénéfique sur les qualités fertilisantes du sol et par conséquent sur le rendement de la culture. La production de matière sèche de manière significative par rapport aux autres traitements suite à l'effet de la fertilisation minérale a été prouvée par Jemali et Soudi (1998). Il est évident que la réutilisation des eaux usées et des boues résiduaires est faite, non plus comme une spéculation intellectuelle, mais comme une nécessité agro-écologique (Aomar, 1994).

\section{Conclusion}

Au terme de cette étude sur l'évaluation de la production de Moringa oleifera sur boue de vidange dans une perspective de développement durable, les résultats ont montré que le Moringa oleifera a fait preuve d'adaptation aux différents substrats appliqués. Un important taux de survie sur l'ensemble des substrats avec la dose d'irrigation de $1 \mathrm{~L}$ a été enregistré. Les plants issus des substrats BS, BSE et BSC ont donné les meilleurs résultats sur la plupart des paramètres évalués, alors que les plants issus du substrat boue uniquement ont enregistré les plus forts taux de létalité. Cependant, les meilleurs résultats ont été obtenus avec la boue mélangée au compost. En effet, l'apport du compost et d'engrais chimiques ont permis d'avoir des différences sur la croissance et le développement de la plante contrairement à celui de la boue uniquement. Cette boue pourrait donc être utilisée en agriculture comme fertilisant en la supplémentant de fertilisants organiques pour obtenir un bon rendement. En perspective, il serait souhaitable de poursuivre l'étude sur la boue avec d'autres spéculations d'intérêt économique. 


\section{CONFLIT D'INTERETS}

Les auteurs déclarent qu'il n y'a pas de conflit d'intérêts.

\section{CONTRIBUTIONS DES AUTEURS}

Ce travail a été réalisé en collaboration entre tous les auteurs. SSI a conçu le projet et rédigé l'article. MMA a participé à la rédaction du projet et la correction de l'article. ATDB a porté des observations sur le travail et participé à la correction de l'article. ZAB a suivi l'expérimentation sur le terrain et participé à la rédaction de l'article. Tous les auteurs ont lu et approuvé le manuscrit final.

\section{REMERCIEMENTS}

Les auteurs remercient la Faculté d'Agronomie de l'Université Abdou Moumouni, La Société d'Exploitation des Eaux du Niger (SEEN) et la Société de Patrimoine des Eaux du Niger (SPEN) pour l'intérêt accordé à la réalisation de cette étude.

\section{REFERENCES}

Abasse T, Maigachi I, Habba W, Diallo D. 2017. Effet de la supplémentation de la farine des feuilles de Moringa oleifera Lam., dans la production des poulets de chair au Niger. Int. J. Biol. Chem. Sci., 11(2): $\quad 722-729 . \quad$ DOI: https://dx.doi.org/10.4314/ijbcs.v11i2.15

ADEME. 2011. Collecte, tri, recyclage et valorisation des déchets. Feuille de Route Stratégique, ADEME, France, 28 p.

Agroconsult H. 2016. Analyse des Potentialités de l'Exploitation du Moringa en Haïti. Ministère de l'Agriculture, des Ressources Naturelles et du Développement Rural (MARNDR), Banque de la République d'Haïti (BRH), Rezo Moringa Dolivayiti, Haïti, 187 p.

Amaglo NK, Timpo GM, Ellis WO, Bennett RN. 2006. Effet de l'écartement et la fréquence des récoltes sur la croissance et le rendement en feuilles de Moringa oleifera Lam. et autres végétaux à fort potentiel nutritionnel: stratégies, normes et marchés pour un meilleur impact sur la nutrition en Afrique. Conférence d'Accra, 16-18 novembre 2006, Ghana, p.11.
AMORCE. 2012. Boues de station d'épuration : technique, valorisation et élimination. Série Technique- DT51 de l'AMORCE, France, $36 \mathrm{p}$.

Anwar F, Bhanger MI. 2003. Analytical characterization of Moringa oleifera seed oil grown in temperate regions of Pakistan. J. Agric. Food Chem., 51(22): 6558- 6563. DOI: $10.1021 /$ jf0209894

Aomar B. 1994. Caractérisation et valorisation agricole des boues résiduaires de Draa Ben Khedda (Algérie). Cahiers Agricultures, 3: 295-299.

APRIFEL. 2001. Les boues d'épuration. Document de synthèse, Comité Sécurité Alimentaire, France, 43 p.

Bosch CH. 2011. Moringa sp. (Néverdier). FORMAD Environment PROTA Network Office Europe, Wageningen University, Gelderland, $34 \mathrm{p}$.

Chouial M, Ben Amirouche S, Belbeldi O. 2017. Performances et limites d'utilisation des boues des stations d'épuration pour l'élevage de plants forestiers en pépinière : Cas du pin maritime (Pinus pinaster Ait.). Revue Agriculture, 8(1): 55 - 67.

CUSSTR. 2008. Gestion des déchets. Commission Universitaire de Sécurité et Santé au Travail Romande, Suisse, 84 p.

De-Saint-Sauveur A, Broin M. 2010. Produire et transformer les feuilles de Moringa. Moringa news / Moringa Association of Ghana, Ghana, 69 p.

Farid L, Sonia A. 2016. Perspectives de valorisation agricole et énergétique des boues issues des STEP en Algérie. Office National de l'Assainissement, Algérie, 58 p.

Foidl N, Makkar HPS, Becker K. 2001. Potentiel de Moringa oleifera en agriculture et dans l'industrie. Potentiel de développement des produits du Moringa, Dar es Salaam, Tanzanie, 20 p. Haouvang LC, Ngakou A, Yemefack M, Mbailao M. 2017. Growth response of Moringa oleifera Lam. as affected by various amounts of compost under greenhouse conditions. Annals of Agricultural Sciences, 62: 221-226. DOI: 
https://doi.org/10.1016/j.aoas.2017.12.00 4

Igoud S. 2001. Valorisation des boues résiduaires issues des stations d'epuration urbaines par leur epandage dans les plantations forestières. Rev. Energ. Ren. : Production et Valorisation-Biomasse, 9: 69-74.

Jemali A, Soudi B, Berdai H. 1998. Valorisation agricole des boues résiduaires: valeur fertilisante et leur impact sur les sols. In Proceeding of 13th International Congress on Agricultural Engineering: Land and Water Use, Rabat, Maroc, $16 \mathrm{p}$.

Koul B, Chase N. 2015. Moringa oleifera Lam.: Panacea to several maladies. Journal of Chemical and Pharmaceutical Research, 7(6): 687-707.

Laurence R. 2017. Valorisation des légumesFeuilles et introduction de Moringa oleifera chez les maraîchers de Toliara. Mémoire de diplôme d'études approfondies (DEA) en Biodiversité et Environnement, Option Biologie végétale, Université de Toliara, Madagascar, $63 \mathrm{p}$.

Madi O, Sali B, Woin N. 2012. Utilisations et importances socio-économiques du Moringa oleifera Lam. en zone de savanes d'Afrique Centrale. Cas de la ville de Maroua au Nord-Cameroun. Journal of Applied Biosciences, 60: 4421- 4432.

Malo T. 2014. Effet de la fertilisation sur la croissance et la production de Moringa oleifera local et Moringa oleifera PKM-1 dans la Région des Cascades. Mémoire de fin de cycle pour l'obtention du diplôme de Master en productions végétales. Université Polytechnique de BoboDioulasso, Burkina Faso, 68 p.

Martin LP. 2007. The Moringa tree. Educational Concerns for Hunga Organization (ECHO) Technical Note, USA, $22 \mathrm{p}$.

Morana J. 2005. La gestion des déchets : d'une logique de traiteur à une logique d'expert. Exemple de la société SITA, Institut d'Administration des Entreprises,
Document de recherche $\mathrm{N}^{\circ}$ 2005-04, France, $16 \mathrm{p}$.

Pamo ET, Boukila B, Tendonkeng F. 2002. Influence de la fumure organique, du NPK et du mélange des deux fertilisants sur la croissance de Moringa oleifera Lam. dans l'Ouest du Cameroun. IRAD, Cameroun, $13 \mathrm{p}$.

Phatu WM. 2019. Responses of Moringa oleifera root growth to container size during overwintering in temperate regions. African Journal of Agricultural Research, 14(2): 65-68. DOI: 10.5897/AJAR2016.11667

Pisson C. 2000. Impact de l'épandage agricole de boues résiduaires urbaines sur la qualité des productions céréalières en particulier sur l'aspect des éléments traces métalliques. Mémoire de fin d'études, Formation des ingénieurs du génie sanitaire, Ecole Nationale de la Santé Publique (ENSP_RENNES), France, 102 p.

Sattah S, Haloui F. 2019. Effet du plomb sur les paramètres morpho-physiologiques de Moringa oleifera Lam. Mémoire de fin d'étude en vue de l'obtention du diplôme de Master en biologie, Spécialité Biodiversité et environnement, Université Abdelhamid Ibn Badis Mostaganem, Algérie, $52 \mathrm{p}$.

Vorburger J. 2006. Ecologie industrielle et valorisation des déchets. MBA Gestion Internationale, Faculté des Sciences de l'Administration, Université LAVAL, Canada, $158 \mathrm{p}$.

Yansoumba B. 2002. Possibilités d'utilisation des eaux usées dans la production forestière : cas de Leptadenia hastata et de Moringa oleifera. Mémoire de fin d'études, ITA, Faculté d'Agronomie de l'Université Abdou Moumouni de Niamey, Niger, $37 \mathrm{p}$.

Zakaria OO. 2013. Essai de culture de Moringa oleifera en pot sur sols argileux et sableux. Mémoire de fin de cycle pour l'obtention de la Licence générale, Faculté d'Agronomie, Université Abdou Moumouni de Niamey, Niger, 56 p. 